\title{
Investigação de mudanças do status salino do solo pelo emprego de análise multivariada
}

\author{
Eunice M. Andrade ${ }^{1}$, Ana. C. M. Meireles ${ }^{1}$, Deborah M. B. Alexandre ${ }^{2}$, \\ Helba A. Q. Palácio ${ }^{3} \&$ Cleene A. Lima ${ }^{3}$
}

\begin{abstract}
RESUMO
O bjetivou-se investigar a aplicabilidade da técnica de estatística multivariada, análise de agrupamento, como ferramenta para identificar a similaridade nas concentrações de sais em campos irrigados. A pesquisa foi desenvolvida em três áreas, sendo uma $\mathrm{M}$ ata $\mathrm{N}$ ativa e duas irrigadas do Distrito de Irrigação Jaguaribe-A podi (DIJA), nos municípios de Limoeiro do N orte e Q uixeré, Ceará. As coletas de solo foram realizadas mensalmente, de dezembro/1999 a dezembro/2000 e nos meses de setembro a dezembro de 2001, nas profundidades de $0-30$ e $30-60 \mathrm{~cm}$. Foram considerados os seguintes atributos: CEes (condutividade elétrica do extrato de saturação do solo), $\mathrm{Ca}^{2+}+\mathrm{M} \mathrm{g}^{2+}, \mathrm{Cl}^{\prime}, \mathrm{Na}^{+}$e K${ }^{+}$e a RAS (razão de adsorção do sódio). A aplicação da análise de agrupamento resultou em três grupos similares quanto aos atributos estudados, havendo diferença significativa $(p<0,001)$ entre os valores representativos da $\mathrm{M}$ ata $\mathrm{N}$ ativa e aqueles dos dois campos irrigados. A técnica de análise de agrupamento mostrou-se como ferramenta apropriada para definir a semelhança entre os atributos estudados, independentemente da sua posição no tempo ou no espaço. Tal fato expressa a aplicabilidade da mesma em estudos de identificação de áreas similares com maiores ou menores risco de salinidade.
\end{abstract}

Palavras-chave: salinidade, semiárido, análise de agrupamento, irrigação-impacto

\section{Investigation of the changes in the status of soil salinity using multivariable analysis}

\begin{abstract}
This w ork was carried out to investigate the multivariable statistics/cluster analysis as a tool to identify the similarity of irrigated fields. The investigation was conducted in three areas. O ne was an undisturbed native area (MN) and the others, two irrigated field in the Irrigated District of Jaguaribe-Apodi (DIJA) situated in the municipality of Limoeiro do Norte and Quixeré, Ceará State, Brazil. Soil was sampled monthly, from December/1999 to December/2000, and also from September to December of 2001 in the depths of $0-30,30-60 \mathrm{~cm}$. The attributes considered in this study were: EC (electric conductivity of soil solution), $\mathrm{Ca}^{2+}+\mathrm{Mg}^{2+}, \mathrm{Cl}^{-}, \mathrm{Na}^{+}$and $\mathrm{K}^{+}$and $\mathrm{SAR}$ (sodium adsorption ratio). The Cluster Analysis application identified the similarity of studied attributes and three homogeneous groups were identified. The studied attributes values from M N were statistically different $(p<0.001)$ from those of irrigated fields. According to the results, Cluster Analysis showed up as a suitable tool to define the similarity independent of sample position in time and space. This fact expresses that Cluster Analysis technique can be applied to identify the similarity in areas with high or little salinization risk.
\end{abstract}

Key words: salinity, semiarid, cluster analysis, irrigation-impact

Trabalho submetido e selecionado no primeiro Simpósio Brasileiro de Salinidade realizado de 12-15/10/2010 em Fortaleza, Ceará, Brasil ${ }^{1}$ DEN A/UFC, Av. Mister Hull, S/N , Caixa Postal 12.168, Campus do Pici, CEP 60455-970, Fortaleza, CE, Fone: (85) 3366-9762. E-mail: eandrade@pq.cnpq.br; ameireles2003@yahoo.com.br

${ }^{2}$ CO GERH, Rua Adualdo Batista, 1550, Parque Iracema, CEP 60824-140, Fortaleza, CE, Fone (85) 3218-7653. E-mail: deborah.barros@cogerh.com.br 3 IFCE,Campus Iguatu-CE, Rodovia Iguatu/N. Alegre Km 05, S/N, Caixa Postal 38, Vila Cajazeiras, CEP 63500-000, Iguatu, CE, Fone (88) 35821000, ramal 252. E-mail: helbaraujo23@yahoo.com.br; cleene2@hotmail.com 


\section{INTRODUÇÃO}

No início da Era Cristã ocorreu o declínio das áreas agrícolas irrigadas nos vales dos rios Tigre e Eufrates, em decorrência da salinidade do solo, tornando terras férteis em áreas improdutivas. Este impacto desencadeou o êxodo humano das regiões secas para as úmidas e a irrigação perdeu sua função vital, passando a desempenhar função secundária

O crescimento demográfico de forma contínua e exponencial conduziu a humanidade a resgatar a prática da agricultura irrigada, seja como técnica de suplementação das chuvas nas regiões úmidas seja como uma técnica para tornar os solos das zonas áridas e semiáridas do globo produtivos. No entanto, os mesmos problemas de salinidade que levaram a irrigação ao declínio há milhares de anos, continuam a ser identificados nas áreas irrigadas das mais diferentes partes do globo (D' Almeida, 2002; Burkhalter \& Gates, 2006; Andrade, 2009; Quinn, 2009).

Atualmente, estima-se uma extensão de 270 milhões de hectares irrigados, o que corresponde a apenas $15 \%$ da terra cultivada, mas responde por $40 \%$ da produção de fibras e alimentos do planeta (Tyler, 2007). Desses 270 milhões de hectares, 160 estão situados em regiões de clima úmido, os outros 110 milhões estão localizados em zonas secas cujo total precipitado não é suficiente para promover a lixiviação dos sais adicionados ao solo durante o período de irrigação, aumentando o risco de acúmulo de sais no solo (Smedema \& Shiati, 2002; Andrade et al., 2004; Fernandes et al., 2005).

A condição de baixas alturas pluviométricas registradas nas regiões semiáridas é agravada pelo elevado saldo de energia solar disponível ao processo de evapotranspiração. A combinação desses dois processos naturais com a adoção da irrigação resulta em altas concentrações de sais no solo (Meireles et al., 2003; Brito et al., 2005; Chaves et al., 2006; Lopes et al., 2008). A mudança das taxas de evapotranspiração nessas regiões pela adoção da irrigação, mesmo em uma visão conservacionista, resulta em uma adição de 3 a $5 \mathrm{t} \mathrm{ha} \mathrm{ano}^{-1} \mathrm{de}$ sais ao solo explorado pela agricultura irrigada (Aragués \& Tanji, 2003; Causapé et al., 2004).

Ante esta condição torna-se imprescindível o conhecimento do status salino no solo e na água, bem como os sais que estão se acumulando, uma vez que os mesmos atuam diferentemente sobre a estrutura do solo. No monitoramento de sais se adotam medidas de múltiplos parâmetros, as quais são efetuadas em diferentes épocas e originadas de diferentes áreas, gerando uma complexa matriz multivariada de difícil interpretação, decorrente do grande número de informações interdependentes contidas na mesma.

Há mais de duas décadas que pesquisadores de diferentes partes do mundo (Nathan \& MCmahon, 1990; Mohan \& Arumugam, 1996; Singh et al., 2004) vêm empregando técnicas de estatística multivariada nas ciências naturais para auxiliar na interpretação de matrizes geradas pelo monitoramento dos processos naturais multivariados. Recentemente, pesquisadores como Palácio (2004); Andrade et al. (2008); Meireles et al. (2010) e Palácio et al. (2009) iniciaram a aplicação de técnicas de estatística multivariada - análise de agrupamento hierárquico e análise fatorial/análise de componentes principais na identificação de grupos similares e dos fatores determinantes da qualidade das águas em áreas irrigadas no estado do Ceará.

Portanto, este trabalho tem como objetivo investigar a aplicabilidade da técnica de estatística multivariada, análise de agrupamento, como ferramenta para identificar a similaridade das áreas com maiores ou menores concentrações de sais em campos irrigados no Semiárido cearense.

\section{Material e MÉTODOS}

A pesquisa foi desenvolvida no período de dezembro de 1999 a dezembro de 2001 em três áreas, sendo um campo não trabalhado (Mata Nativa) e dois explorados com a cultura da banana irrigada no Distrito de Irrigação Jaguaribe-Apodi (DIJA), nos municípios de Limoeiro do Norte e Quixeré. As áreas estão localizadas entre as coordenadas geográficas $05^{\circ}$ $06^{\prime} 38^{\prime \prime}$ e $05^{\circ} 11^{\prime} 39^{\prime \prime}$ de Latitude Sul e $37^{\circ} 52^{\prime} 21^{\prime \prime}$ e $37^{\circ} 56^{\prime} 05^{\prime \prime}$ de Longitude oeste.

A região apresenta clima quente e semiárido, BSw'h', com chuvas máximas de outono e temperatura média mensal sempre superior a $18{ }^{\circ} \mathrm{C}$. A pluviosidade média da região é de $750 \mathrm{~mm}$, sendo que o período mais seco é o de julho a dezembro, a evaporação potencial média anual é de $3.215 \mathrm{~mm}$ e a umidade relativa média anual é de $62 \%$. Para o período de estudo o total precipitado nas duas estações chuvosas foi de 1.257 e 566,7 $\mathrm{mm}$, nos anos de 2000 e 2001, respectivamente (Figura 1). Os solos são classificados como Cambissolos Háplicos (EMBRAPA, 2006).

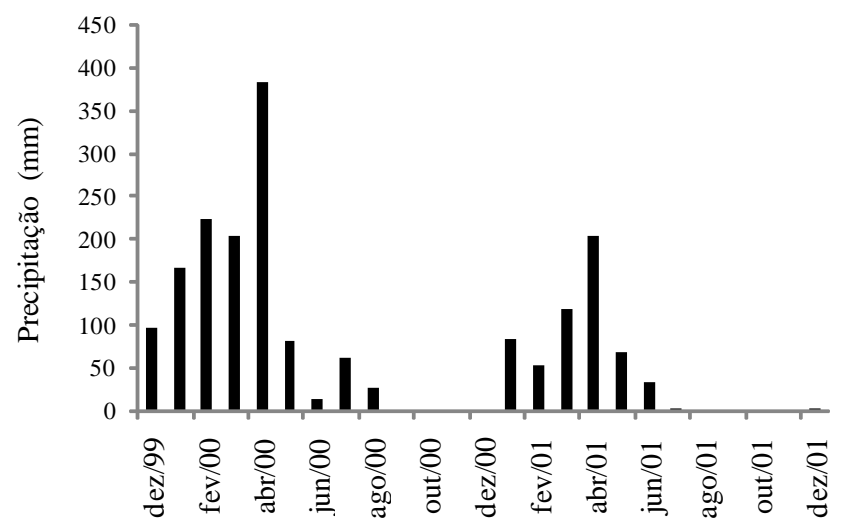

Figura 1. Distribuição dos totais mensais precipitados na Chapada do A podi, Ceará no período de dez/1999 a dez/ 2001

As águas utilizadas nos dois campos irrigados são oriundas de fontes distintas, águas superficiais e águas subterrâneas (Tabela 1). Na região de Limoeiro do Norte se emprega as águas do rio Jaguaribe enquanto no município de Quixeré a fonte hídrica são poços com profundidade média de $100 \mathrm{~m}$.

As coletas de solo, para as três áreas foram realizadas mensalmente no período de dezembro/1999 a dezembro/2000 e nos meses de setembro, outubro, novembro e dezembro de 2001 , nas profundidades de 0-30 e 30-60 cm. As análises físicoquímicas foram realizadas no Laboratório de Análise de Solos e Água para fins de Irrigação do CENTEC, de Limoeiro do 
Tabela 1. Q ualidade das águas superficiais e subterrâneas utilizadas na irrigação em Limoeiro do N orte e em Q uixeré, Ceará, período de dezembro/1999 a dezembro/2000

\begin{tabular}{|c|c|c|c|c|c|c|c|c|c|c|c|c|c|}
\hline Parâmetros & Dez & Jan & Fev & Mar & Abr & Maio & Jun & Jul & Ago & Set & Out & Nov & Dez \\
\hline & \multicolumn{13}{|c|}{ Água de superfície (Limoeiro do Norte) } \\
\hline $\mathrm{CE}\left(\mathrm{dS} \mathrm{m}^{-1}\right)$ & 0,33 & 0,28 & 0,32 & 0,20 & 0,27 & 0,31 & 0,39 & 0,28 & 0,33 & 0,37 & 0,36 & 0,37 & 0,28 \\
\hline $\mathrm{pH}$ & 8,53 & 9,15 & 8,67 & 8,23 & 8,60 & 8,03 & 8,36 & 8,36 & 8,46 & 8,21 & 8,51 & 8,21 & 8,36 \\
\hline $\mathrm{Ca}^{2+}\left(\mathrm{mmol}_{\mathrm{c}} \mathrm{L}^{-1}\right)$ & 0,44 & 1,00 & 0,72 & 0,72 & 0,76 & 1,16 & 1,12 & 0,76 & 0,72 & 0,76 & 0,84 & 0,82 & 0,76 \\
\hline $\mathrm{Mg}^{2+}\left(\mathrm{mmol}_{\mathrm{c}} \mathrm{L}^{-1}\right)$ & 1,10 & 2,12 & 0,90 & 0,80 & 1,40 & 1,04 & 1,12 & 0,76 & 0,80 & 0,92 & 1,24 & 0,92 & 0,76 \\
\hline $\mathrm{Na}^{+}\left(\mathrm{mmol}_{\mathrm{c}} \mathrm{L}^{-1}\right)$ & 1,53 & 0,65 & 1,88 & 0,98 & 1,21 & 1,37 & 1,74 & 1,52 & 1,57 & 1,74 & 2,00 & 1,74 & 1,52 \\
\hline $\mathrm{K}^{+}\left(\mathrm{mmol}_{\mathrm{c}} \mathrm{L}^{-1}\right)$ & 0,14 & 0,22 & 0,13 & 0,12 & 0,14 & 0,18 & 0,26 & 0,20 & 0,23 & 0,27 & 0,29 & 0,27 & 0,20 \\
\hline $\mathrm{Cl}^{-}\left(\mathrm{mmol}_{\mathrm{c}} \mathrm{L}^{-1}\right)$ & 1,47 & 1,33 & 1,61 & 0,93 & 1,35 & 1,38 & 1,95 & 1,30 & 1,13 & 1,52 & 1,35 & 1,52 & 1,30 \\
\hline $\mathrm{CO}_{3}^{-}\left(\mathrm{mmol}_{\mathrm{c}} \mathrm{L}^{-1}\right)$ & 0,32 & 1,00 & 0,24 & 0,08 & 0,08 & 0,08 & 0,16 & 0,08 & 0,16 & 0,14 & 0,16 & 0,14 & 0,08 \\
\hline $\mathrm{HCO}_{3}^{-}\left(\mathrm{mmol}_{\mathrm{c}} \mathrm{L}^{-1}\right)$ & 3,56 & 0,86 & 1,61 & 1,38 & 1,30 & 1,90 & 1,93 & 1,67 & 1,79 & 2,09 & 2,21 & 2,09 & 1,67 \\
\hline $\operatorname{RAS}\left(\mathrm{mmol}_{\mathrm{c}} \mathrm{L}^{-1}\right)^{0,5}$ & 1,54 & 0,86 & 1,74 & 0,93 & 1,00 & 1,06 & 1,34 & 1,42 & 1,48 & 1,58 & 1,66 & 1,54 & 1,42 \\
\hline Classe & $\mathrm{C}_{2} \mathrm{~S}_{1}$ & $\mathrm{C}_{2} \mathrm{~S}_{1}$ & $\mathrm{C}_{2} \mathrm{~S}_{1}$ & $\mathrm{C}_{1} \mathrm{~S}_{1}$ & $\mathrm{C}_{2} \mathrm{~S}_{1}$ & $\mathrm{C}_{2} \mathrm{~S}_{1}$ & $\mathrm{C}_{2} \mathrm{~S}_{1}$ & $\mathrm{C}_{2} \mathrm{~S}_{1}$ & $\mathrm{C}_{2} \mathrm{~S}_{1}$ & $\mathrm{C}_{2} \mathrm{~S}_{1}$ & $\mathrm{C}_{2} \mathrm{~S}_{1}$ & $\mathrm{C}_{2} \mathrm{~S}_{1}$ & $\mathrm{C}_{2} \mathrm{~S}_{1}$ \\
\hline \multicolumn{14}{|c|}{ Água de Poço (Quixeré) } \\
\hline $\mathrm{CE}\left(\mathrm{dS} \mathrm{m}^{-1}\right)$ & 1,78 & 1,83 & 1,79 & 1,84 & 1,82 & 1,83 & 1,87 & $* *$ & 1,88 & 1,94 & 1,87 & 1,83 & 1,88 \\
\hline $\mathrm{pH}$ & 6,73 & 7,05 & 6,89 & 6,73 & 6,91 & 7,12 & 6,99 & $* *$ & 7,10 & 7,79 & 7,05 & 6,98 & 6,97 \\
\hline $\mathrm{Ca}^{2+}\left(\mathrm{mmol}_{\mathrm{c}} \mathrm{L}^{-1}\right)$ & 16,84 & 7,16 & 7,10 & 6,68 & 6,16 & 7,36 & 5,52 & $* *$ & 8,88 & 9,00 & 8,40 & 7,40 & 8,02 \\
\hline $\mathrm{Mg}^{2+}\left(\mathrm{mmol}_{\mathrm{c}} \mathrm{L}^{-1}\right)$ & 5,82 & 5,73 & 3,64 & 3,48 & 4,96 & 4,40 & 5,64 & $* *$ & 4,64 & 3,32 & 3,92 & 5,96 & 3,50 \\
\hline $\mathrm{Na}^{+}\left(\mathrm{mmol}_{\mathrm{c}} \mathrm{L}^{-1}\right)$ & 4,81 & 1,65 & 8,53 & 5,53 & 5,23 & 5,39 & 5,44 & $* *$ & 5,10 & 5,69 & 5,92 & 2,93 & 6,15 \\
\hline $\mathrm{K}^{+}\left(\mathrm{mmol}_{\mathrm{c}} \mathrm{L}^{-1}\right)$ & 0,14 & 0,07 & 0,03 & 0,05 & 0,06 & 0,06 & 0,07 & $* *$ & 0,07 & 0,07 & 0,07 & 0,07 & 0,07 \\
\hline $\mathrm{Cl}^{-}\left(\mathrm{mmol}_{\mathrm{c}} \mathrm{L}^{-1}\right)$ & 10,32 & 10,49 & 10,26 & 10,74 & 10,89 & 10,72 & 10,15 & $* *$ & 10,46 & 14,38 & 11,20 & 10,58 & 10,43 \\
\hline $\mathrm{CO}_{3}^{-}\left(\mathrm{mmol}_{\mathrm{c}} \mathrm{L}^{-1}\right)$ & ND & ND & ND & ND & ND & ND & ND & $* *$ & ND & ND & ND & ND & ND \\
\hline $\mathrm{HCO}_{3}^{-}\left(\mathrm{mmol}_{\mathrm{c}} \mathrm{L}^{-1}\right)$ & 10,84 & 5,40 & 5,86 & 5,48 & 5,36 & 5,20 & 5,20 & $* *$ & 5,10 & 5,20 & 6,28 & 6,44 & 6,80 \\
\hline RAS $\left(\mathrm{mmol}_{\mathrm{c}} \mathrm{L}^{-1}\right)^{0,5}$ & 1,08 & 0,52 & 2,85 & 1,91 & 1,78 & 1,74 & 1,88 & $* *$ & 1,52 & 1,74 & 1,84 & 0,91 & 1,97 \\
\hline Classe* & $\mathrm{C}_{3} \mathrm{~S}_{1}$ & $\mathrm{C}_{3} \mathrm{~S}_{1}$ & $\mathrm{C}_{3} \mathrm{~S}_{1}$ & $\mathrm{C}_{3} \mathrm{~S}_{1}$ & $\mathrm{C}_{3} \mathrm{~S}_{1}$ & $\mathrm{C}_{3} \mathrm{~S}_{1}$ & $\mathrm{C}_{3} \mathrm{~S}_{1}$ & $* *$ & $\mathrm{C}_{3} \mathrm{~S}_{1}$ & $\mathrm{C}_{3} \mathrm{~S}_{1}$ & $\mathrm{C}_{3} \mathrm{~S}_{1}$ & $\mathrm{C}_{3} \mathrm{~S}_{1}$ & $\mathrm{C}_{3} \mathrm{~S}_{1}$ \\
\hline
\end{tabular}

ND não detectado; * classificação de Richards (1994); ** coleta não realizada

Norte sendo considerados, neste estudo, os seguintes atributos: CEes (Condutividade Elétrica do extrato de saturação do solo), $\mathrm{Ca}^{2+}+\mathrm{Mg}^{2+}, \mathrm{Cl}^{-}, \mathrm{Na}^{+}$e K${ }^{+}$e a RAS (razão de adsorção do sódio). As análises resultaram em uma matriz de 6 colunas (atributos) e 96 linhas (número de coleta x camadas estudadas) totalizando 576 entradas.

Para se identificar a similaridade da dinâmica dos íons no tempo e no espaço, utilizou-se a análise de agrupamento pelo método de agrupamento hierárquico via processamento no software SPSS 16.0. Uma vez que a similaridade entre as variáveis é dependente da escala e das unidades em que as mesmas são expressas (Dillon \& Goldstein, 1984), elas devem ser padronizadas antes de serem operadas. Neste estudo, para se evitar os erros devidos às escalas e unidades das variáveis selecionadas, as mesmas tiveram seus escores normalizados para o padrão $Z(\mu=0 ; \sigma=1)$.

A similaridade entre os atributos investigados foi estimada por meio da distância Euclidiana ao quadrado, enquanto a definição dos agregados foi efetuada empregando-se o algoritmo de Ward. O ponto de corte para definição dos grupos similares seguiu o preceito apresentado por Hair et al. (2005), ou seja, o mesmo será realizado na primeira grande diferença entre os coeficientes de aglomeração (CA), calculado pela Eq. 1, partindo-se do princípio de que aumentos repentinos no valor do coeficiente de aglomeração representam fusão de elementos diferentes, aparecendo no dendrograma como maiores distâncias (Dillon \& Goldstein, 1984).

$$
C A=\sum_{j=1}^{k}\left(\sum_{i=1}^{n_{j}} X_{i j}^{2}-\frac{1}{n_{j}}\left(\sum_{i=1}^{n_{j}} X_{i j}\right)^{2}\right)
$$

onde:

CA - coeficiente de aglomeração

$\mathrm{X}_{\mathrm{ij}}$ - valor da amostra i individual em cada grupo $\mathrm{j}$

$\mathrm{K}$ - total de amostras em cada estágio

$\mathrm{n}_{\mathrm{j}}$ - número de amostras do grupo

Os dados médios de cada parâmetro analisado dos respectivos grupos formados foram submetidos à análise de teste de média e confrontados pelo teste t a 0,01 de probabilidade de erro, através do programa SPSS 16.0 for Windows.

\section{RESULTADOS E DISCUSSÃO}

O emprego da análise multivariada/análise de agrupamento na investigação da similaridade entre os íons presentes nos solos irrigados da Chapada do Apodi e da Mata Nativa resultou em um arranjo de três grupos homogêneos das 576 entradas da matriz original. $\mathrm{O}$ corte no dendrograma para definir o número de grupos similares foi efetuado quando surgiu a primeira grande diferença da distância reescalonada (Figura 2), a qual expressa a primeira ocorrência de uma dissimilaridade maior entre os coeficientes de aglomeração de dois grupos consecutivos (Palácio et al., 2009).

O dendrograma mostra claramente que a similaridade da salinidade do solo para as áreas estudadas foi definida pela variabilidade espacial das concentrações dos atributos investigados $\left(\mathrm{Ca}^{2+}+\mathrm{Mg}^{2+}, \mathrm{Cl}^{-}, \mathrm{Na}^{+}, \mathrm{K}^{+}\right.$e a RAS $)$, não ocorrendo uma influência maior da variabilidade temporal, uma vez que os arranjos ocorreram de forma independente da data de amostragem. 
Reescalonamento da distância de agrupamento

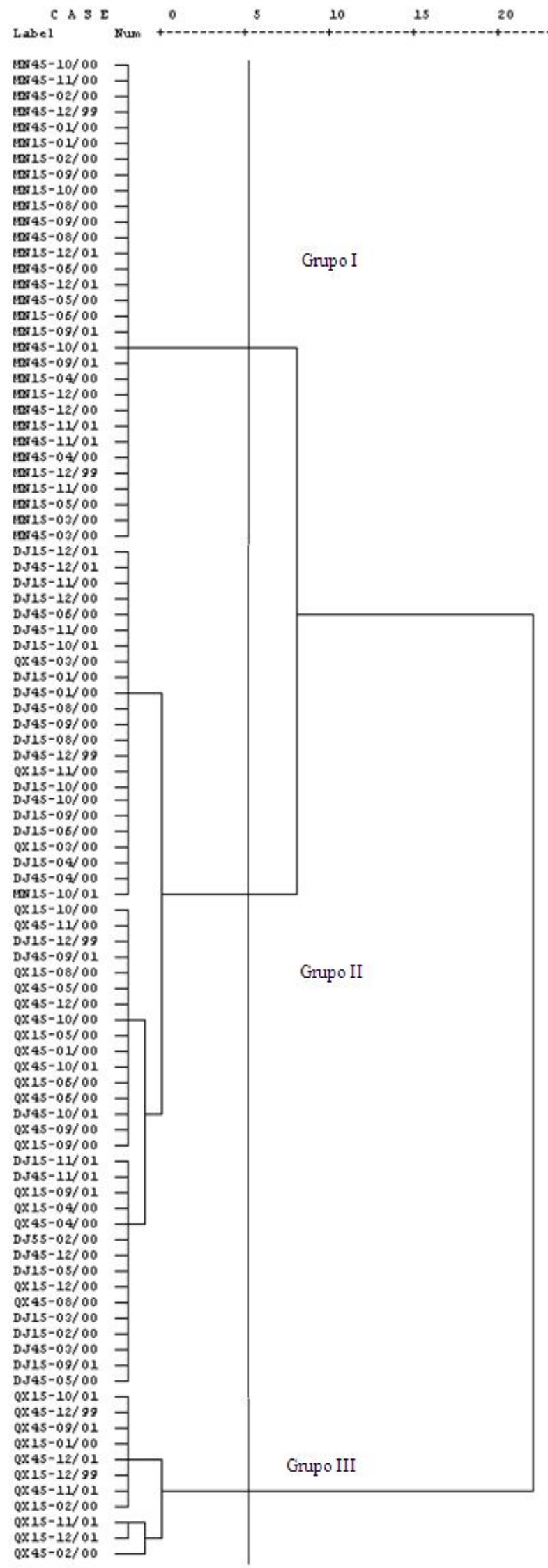

Figura 2. Dendrograma das amostras de solo agrupadas quanto à concentração iônica em áreas de mata nativa e camposirrigados na Chapada do Apodi, Ceará
O grupo I foi composto unicamente pelas concentrações iônicas oriundas de solos da Mata Nativa, independentemente da profundidade amostrada e da época de coleta. O dendrograma expressa a alta similaridade entre os casos que compõem o grupo I (Figura 2). Esta homogeneidade é identificada pelo fato de que as ligações entre eles ocorreram simultaneamente e com o menor registro na diferença do coeficiente de aglomeração, o qual é expresso pelo menor valor da distância de similaridade reescalonada (Hair et al., 2005).

Todos os atributos investigados foram determinantes na definição do grupo I - Mata Nativa, uma vez que suas médias diferiram dos demais grupos ao nível de $1 \%$ de significância (Tabela 2). Observa-se, pela referida tabela, que as

Tabela 2. M édia, valores máximo e mínimo dos atributos químicos em estudo para os grupos do solo na Chapada do Apodi, Ceará, definidos pela técnica da análise de agrupamento

\begin{tabular}{|c|c|c|c|c|}
\hline Atributos & Estatísticas & Grupo 1 & Grupo 2 & Grupo 3 \\
\hline $\begin{array}{c}C E \\
\left(\mathrm{dS} \mathrm{m}^{-1}\right)\end{array}$ & $\begin{array}{c}\mathrm{P}_{5 \%} \\
\mathrm{P}_{95 \%} \\
\text { Mínimo } \\
\text { Máximo } \\
\text { Média } \\
\text { SD } \\
\text { CV }\end{array}$ & $\begin{array}{c}0,07 \\
0,97 \\
0,02 \\
0,45 \\
0,19 \text { a } \\
0,08 \\
42,11\end{array}$ & $\begin{array}{c}0,55 \\
2,27 \\
0,54 \\
3,75 \\
1,17 \mathrm{~b} \\
0,58 \\
49,57\end{array}$ & $\begin{array}{c}0,77 \\
4,22 \\
2,15 \\
4,90 \\
3,21 \mathrm{c} \\
0,92 \\
28,66\end{array}$ \\
\hline $\begin{array}{c}\text { RAS } \\
\left(\mathrm{mmol} \mathrm{L}^{-1}\right)^{1 / 2}\end{array}$ & $\begin{array}{c}P_{5 \%} \\
P_{95 \%} \\
\text { Mínimo } \\
\text { Máximo } \\
\text { Média } \\
\text { SD } \\
\text { CV }\end{array}$ & $\begin{array}{c}0,24 \\
0,97 \\
0,24 \\
1,12 \\
0,52 \text { a } \\
0,19 \\
36,54\end{array}$ & $\begin{array}{c}0,94 \\
3,88 \\
0,88 \\
5,21 \\
2,30 \mathrm{~b} \\
0,88 \\
38,26\end{array}$ & $\begin{array}{c}1,69 \\
4,22 \\
1,69 \\
4,30 \\
2,73 \mathrm{~b} \\
0,77 \\
28,21\end{array}$ \\
\hline $\begin{array}{c}\mathrm{Ca}^{2+}+\mathrm{Mg}^{2+} \\
\left(\mathrm{mmol}_{\mathrm{c}} \mathrm{L}^{-1}\right)\end{array}$ & $\begin{array}{c}P_{5 \%} \\
P_{95 \%} \\
\text { Mínimo } \\
\text { Máximo } \\
\text { Média } \\
\text { SD } \\
C V\end{array}$ & $\begin{array}{c}0,53 \\
3,56 \\
0,50 \\
4,60 \\
1,44 \text { a } \\
0,85 \\
59,03\end{array}$ & $\begin{array}{c}3,61 \\
15,70 \\
3,00 \\
23,10 \\
7,75 b \\
4,13 \\
53,29\end{array}$ & $\begin{array}{c}12,70 \\
33,12 \\
12,70 \\
33,60 \\
20,93 \mathrm{c} \\
6,43 \\
30,72\end{array}$ \\
\hline $\begin{array}{c}\mathrm{Na}^{+} \\
\left(\mathrm{mmol}_{\mathrm{c}} \mathrm{L}^{-1}\right)\end{array}$ & $\begin{array}{c}P_{5 \%} \\
P_{95 \%} \\
\text { Mínimo } \\
\text { Máximo } \\
\text { Média } \\
\text { SD } \\
C V\end{array}$ & $\begin{array}{c}0,11 \\
0,68 \\
0,05 \\
0,72 \\
0,41 \text { a } \\
0,15 \\
36,59\end{array}$ & $\begin{array}{c}0,87 \\
7,91 \\
0,71 \\
11,96 \\
3,90 \mathrm{~b} \\
2,09 \\
53,59\end{array}$ & $\begin{array}{r}6,98 \\
25,59 \\
6,98 \\
26,00 \\
12,27 \mathrm{c} \\
6,53 \\
53,22\end{array}$ \\
\hline $\begin{array}{c}\mathrm{K}^{+} \\
\left(\mathrm{mmol}_{\mathrm{c}} \mathrm{L}^{-1}\right)\end{array}$ & $\begin{array}{c}P_{5 \%} \\
P_{95 \%} \\
\text { Mínimo } \\
\text { Máximo } \\
\text { Média } \\
\text { SD } \\
\text { CV }\end{array}$ & $\begin{array}{l}0,03 \\
0,34 \\
0,02 \\
0,38 \\
0,15 a \\
0,09 \\
60,00\end{array}$ & $\begin{array}{c}0,04 \\
1,76 \\
0,03 \\
1,99 \\
0,32 \mathrm{~b} \\
0,44 \\
137,50\end{array}$ & $\begin{array}{c}0,04 \\
1,45 \\
0,04 \\
1,51 \\
0,51 \mathrm{~b} \\
0,44 \\
86,27\end{array}$ \\
\hline $\begin{array}{c}\mathrm{Cl}^{-} \\
\left(\mathrm{mmol}_{\mathrm{c}} \mathrm{L}^{-1}\right)\end{array}$ & $\begin{array}{c}P_{5 \%} \\
P_{95 \%} \\
\text { Mínimo } \\
\text { Máximo } \\
\text { Média } \\
\text { SD } \\
\text { CV }\end{array}$ & $\begin{array}{l}1,46 \\
4,60 \\
1,42 \\
5,43 \\
2,21 \mathrm{a} \\
0,85 \\
38,46\end{array}$ & $\begin{array}{c}2,86 \\
13,00 \\
2,12 \\
29,05 \\
7,65 \text { b } \\
4,46 \\
58,30\end{array}$ & $\begin{array}{c}17,20 \\
35,81 \\
17,20 \\
35,96 \\
23,42 \mathrm{c} \\
6,66 \\
28,44\end{array}$ \\
\hline
\end{tabular}

Médias seguidas por letras distintas na mesma linha, diferiram entre si pelo teste $t$ ao nível de significância de $1 \%$. $P_{5 \%}$ e $P_{95 \%}$ - percentiles 
concentrações dos mesmos são muito baixas, com valores inferiores aos outros dois grupos em torno de uma magnitude. Estes resultados mostram que os solos em condições naturais não apresentam nenhum risco de salinidade $\left(\mathrm{CE}=0,19 \mathrm{dS} \mathrm{m}^{-1}\right)$ ou de sodicidade $\left(\mathrm{RAS}=0,52\left(\mathrm{mmol} \mathrm{L}^{-1}\right)^{0,5}\right.$.

Tal fato expressa que as concentrações dos íons na Mata Nativa não apresentam entre si variações estatisticamente significativas com relação ao tempo ou espaço, caracterizando o estado de equilíbrio da mesma. Resultados semelhantes foram constatados por outros pesquisadores em estudos do impacto da irrigação no solo em regiões semiáridas do Nordeste brasileiro (Brito et al., 2005; Chaves et al., 2006).

Dos íons investigados, o cloreto foi o que apresentou maiores concentrações na Mata Nativa com valores variando de 1,42 a 5,43 mmol $\mathrm{L}^{-1}$ com um percentil de $95 \%$ igual a 4,60 mmol $_{c} \mathrm{~L}^{-1}$, ou seja, $95 \%$ das observações apresentam concentrações inferiores a este valor. A presença do íon cloreto nos nossos solos pode ser explicada pela dissolução da halita de origem marinha, proveniente dos sedimentos marinhos ou da deposição de aerossóis marinhos.

Outro ponto a ser considerado para esta maior concentração é o fato do cloreto ser um íon conservativo, de difícil degradação na natureza, de maneira que o total de chuva precipitada na área não é suficiente para promover a lixiviação dos mesmos, ocorrendo, assim, ao longo dos tempos, um acúmulo maior nas camadas mais superficiais do solo (Fernandes et al., 2005; Arruda \& Jardim, 2007).

O grupo II representa todos os casos registrados na área irrigada do DIJA e parte daqueles oriundos do campo irrigado do Quixeré, independente da época e da camada amostrada, e foi composto por valores intermediários entre os grupos I e III, expressando que, embora esteja havendo adição de sais ao solo pelo manejo da irrigação, o mesmo vem ocorrendo a taxas menores do que aquelas registradas pelo grupo III.

Para todos os atributos investigados o grupo II diferiu estatisticamente $(\mathrm{p}<0,01)$ do grupo I (Tabela 2$)$, enquanto com relação ao grupo III a dissimilaridade $(\mathrm{p}<0,01)$ foi definida pelos seguintes parâmetros: $\mathrm{CE}, \mathrm{Ca}^{2+}+\mathrm{Mg}^{2+}, \mathrm{Na}^{+} \mathrm{e} \mathrm{Cl}$. Tal fato caracteriza a existência de uma acumulação de sais altamente significativa nos solos irrigados, expressando que o total precipitado na região não foi suficiente para promover a lixiviação dos sais adicionados pela irrigação (Meireles et al., 2003; Andrade et al., 2004; Lopes et al., 2008).

$\mathrm{O}$ aumento médio da $\mathrm{CE}$ para este grupo em relação à Mata Nativa foi de aproximadamente dez vezes, variando de 0,19 para $1,17 \mathrm{dS} \mathrm{m}^{-1}$, e que $95 \%$ dos valores foram inferiores a 2,27 $\mathrm{dS} \mathrm{m}{ }^{-1}$, apesar de se ter observado valores de $3,75 \mathrm{dS} \mathrm{m}^{-1}$. Dentre os íons investigados as maiores concentrações foram registradas para o íon $\mathrm{Cl}^{-}$atingindo valores de 29,05 $\mathrm{mmol}_{\mathrm{c}} \mathrm{L}^{-1}$ apesar da qualidade de água empregada na irrigação ser classificada como $\mathrm{C}_{2} \mathrm{~S}_{1}$. Estas maiores concentrações são decorrentes do emprego de cloreto de potássio como fonte de potássio para a cultura da bananeira, como já referido por pesquisadores como Fernandes et al. (2005) e D’Almeida (2002).

O grupo III foi composto exclusivamente por informações provenientes das amostras realizadas no campo irrigado do Quixeré, em um total de 11 das 32 observações realizadas nesta área. Pode-se observar, pela Figura 2, que em quase sua totalidade o grupo III foi composto por dados correspondentes ao período seco.

Observou-se, também, uma semelhança maior entre os dois grupos (II e III) formados pelos solos oriundos dos campos irrigados, não havendo significância estatística a nível de 0,01 dos valores apresentados pelas RAS e $\mathrm{K}^{+}$. O grupo III se caracteriza como os solos que acumularam as maiores adições de sais pelo manejo da irrigação. Esses maiores acúmulos podem ser explicados pela qualidade da água empregada na irrigação, $\mathrm{C}_{3} \mathrm{~S}_{1}$, e pelo emprego da fertigação (D'Almeida, 2002).

Destaca-se que, embora na estação chuvosa do ano 2000 o total precipitado $(1.257 \mathrm{~mm})$ tenha sido $62 \%$ superior a média da região, o mesmo não foi suficiente para efetuar a lixiviação dos sais adicionados pelo manejo da irrigação. Constatação do efeito acumulativo de sais em áreas irrigadas de regiões semiáridas vem sendo apresentadas por pesquisas desenvolvidas em diferentes partes do globo (Aragués \& Tanji, 2003; Causapé et al. 2004; Chaves et al., 2006).

\section{Conclusões}

1. A dissimilaridade, altamente significativa ( $\mathrm{p}<0,001)$, expressada entre o campo de Mata Nativa e os irrigados, mostra que estes estão recebendo uma taxa anual de sais superior à lixiviação proporcionada pela irrigação e precipitação, podendo conduzir as referidas áreas a um estado de degradação pela salinização, sendo o quadro mais preocupante apresentado pelos campos irrigados do Quixeré.

2. A técnica de análise de agrupamento mostrou-se como ferramenta apropriada para definir a semelhança entre os atributos estudados, independentemente da sua posição no tempo ou no espaço. Tal fato expressa sua aplicabilidade em estudos de identificação de áreas similares, com maiores ou menores riscos de salinidade.

\section{LITERATURA CITADA}

Andrade, E. M. A irrigação e suas implicações sobre o capital natural em regiões áridas e semi-áridas: Uma revisão. Revista Ceres, v.56, p.390-398, 2009.

Andrade, E. M.; D’ Almeida, D. M. B.; Meireles, A. C. M.; Lemos Filho, L. C. A.; Arruda, F. E. R. Elevação da concentração iônica da solução do solo em áreas irrigadas na Chapada do Apodi, CE. Revista Ciência Agronômica, Fortaleza, v.35, p.916, 2004.

Andrade, E. M.; Palácio, H. A. Q.; Crisóstomo, L. A.; Souza, I. H.; Leão, R. A. O.; Guerreiro, M. J. Land use effects in groundwater composition of an alluvial aquifer (Trussu River, Brazil) by multivariate techniques. Environmental Resource, v.106, p.170-177, 2008.

Aragués, R.; Tanji, K. K. Water quality of irrigation return flows. Encyclopedia of water science. Marcel Dekker Inc., p.502506, 2003. 
Arruda, T. L.; Jardim, W. F. Tratamento de água subterânea contaminada com compostos organoclorados usando ferro elementar e o reagente de fenton. Química Nova, v.30, p.16281632,2007

Brito, L. T. L.; Srinivasan, V. S.; Silva, A. S.; Gheyi, H. R.; Galvão, C. O.; Hermes, L. C. Influência das atividades antrópicas na qualidade das águas da Bacia Hidrográfica de Rio Salitre. Revista Brasileira de Engenharia Agrícola e Ambiental, v.9, p.596-602, 2005.

Burkhalter, J. P.; Gates, T. K. Evaluating regional solutions to salinization and waterloggin in an irrigated river valley. Journal of Irrigation and Drainage Engineering, v.132, p.2130, 2006.

Causapé, J.; Quílez, D.; Aragués, R. Assessment of irrigation and environmental quality at the hydrological basin level II. Salt and nitrate loads in irrigation return flows. Agricultural Water Management, v.70, p.211-228, 2004.

Chaves, L. C. G.; Andrade, E. M.; Crisóstomo, L. A.; Ness, R. L. L.; Lopez, J. F. B. Risco de degradação em solo irrigado do Distrito de Irrigação do Perímetro Araras Norte, Ceará. Revista Ciência Agronômica, v.37, p.292-298, 2006.

D’Almeida, D. M. B. A. Risco de salinização de um Cambissolo na Chapada do Apodi-CE. Fortaleza: UFC, 2002. 72p. Dissertação Mestrado

Dillon, W. R.; Goldstein, M. Multivariate analysis methodos and applications. New York: John Wiley e Sons, 1984. 587p.

EMBRAPA - Empresa Brasileira de Pesquisa Agropecuária. Centro Nacional de Pesquisa de Solos. Sistema Brasileiro de Classificação de Solos. 2.ed. Rio de Janeiro: Embrapa Solos, 2006. 306p.

Fernandes, M. A. B.; Santiago, M. M. F.; Gomes, D. F.; Mendes Filho, J.; Frischkorn, H.; Lima, J. O. G. A origem dos cloretos nas águas subterrâneas na Chapada do Apodi-Ceará. Águas Subterrâneas, v.19, p.25-34, 2005.

Hair, J. F.; Anderson, R. E.; Tatham, R. L.; Black, W. C. Análise multivariada de dados. 1.ed. Porto Alegre: Bookman, 2005. $593 p$.
Lopes, J. F. B.; Andrade, E. M.; Chaves, L. C. G. Impacto da irrigação sobre os solos de perímetros irrigados na Bacia do Acaraú, Ceará, Brasil. EngenhariaAgrícola, v.28, p.34-43, 2008.

Meireles, A. C. M.; Andrade E. M.; Chaves, L. C. G.; Frischkorn, H.; Crisóstomo, LA. A new proposal of the classification of irrigation water. Revista Ciência Agronômica, Fortaleza, v.41, p.349-357, 2010.

Meireles A. C. M.; Andrade, E. M.; Cruz, M. G. M.; Lemos Filho, L. C. A. Avaliação do impacto da fertirrigação em cambissolos na Chapada do Apodi, Ceará. Revista Ciência Agronômica, v.34, p.207-212, 2003.

Mohan, S.; Arumugam, N. Relative importance of meteorogical variables im evapotranspiration: Factor analysis approach. Water Resources Management, v.10, p.1-20, 1996.

Nathan, R. J.; Mcmahon, T. A. Identification of homogeneous regions for purposes of regionalization. Journal of Hydrology, v. 121, p.217-238, 1990.

Palácio, H. A. Q. Índice de qualidade de água na parte baixa da bacia hidrográfica do rio Trussu - CE. Fortaleza: UFC, 2004. 95p. Dissertação Mestrado

Palácio, H. A. Q.; Andrade, E. M.; Lopes, F. B.; Alexandre, D. M. B.; Arraes, F. D. D. Similaridade da qualidade das águas superficiais da Bacia do Curu, Ceará. Ciência Rural, v.39, p.2494-2500, 2009.

Quinn, N. W. T. Environmental decision support system development for seasonal wetland salt management in a river basin subjected to water quality regulation. Agricultural Water Management, v.96, p.247-254, 2009.

Singh, K. P.; Malik, A.; Mohan, D.; Sinha, S. Multivariate statistical techniques for the evaluation of spatial and temporal variations in water quality of Gomti River (Índia) - A case study. Water Research, v.38, p.3980-3992, 2004.

Smedema, L. K.; Shiati, K. Irrigation and salinity: A perspective review of the salinity hazards of irrigation development in the arid zone. Irrigation and Drainage Systems, v.16, p.161-174, 2002.

Tyler, M. G. Ciência ambiental. 11.ed. São Paulo: Thompson Learning, 2007. 501p. 